\title{
Conformal symmetry and the nonlinear Schrödinger equation
}

\author{
Pijush K. Ghosh* \\ Department of Physics, Ochanomizu University, \\ 2-1-1 Ohtsuka, Bunkyo-ku, Tokyo 112-8610, Japan.
}

We show that the width of the wave-packet of a class of generalized nonlinear Schrödinger equations (NLSE) trapped in an arbitrary time-dependent harmonic well in any dimensions is universally determined by the same Hill's equation. This class of generalized NLSE is characterized by a dynamical $O(2,1)$ symmetry in absence of the trap. As an application, we study the dynamical instabilities of the rotating as well as non-rotating Bose-Einstein condensates in one and two dimensions. We also show exact extended parametric resonance in a non-relativistic Chern-Simons theory producing a gauged NLSE.

PACS numbers: 05.45.Yv, 03.75.Fi, 11.15.-q, 03.65.Ge

The nonlinear Schrödinger equation (NLSE) appears in many branches of present-day physics and mathematics [1]. The optical soliton [2], a solution of the NLSE, has even been observed experimentally [3]. The one dimensional NLSE is exactly solvable. There exists several other generalized NLSE in one dimension which are also exactly solvable. However, a very little exact and analytical results are known for higher dimensional generalizations of these models, although they are very much relevant in many branches of modern science. The purpose of this letter is to present an exact, analytical description of the dynamics of the width of the wave-packet for a class of generalized NLSE in arbitrary dimensions trapped in a time-dependent harmonic well. This class of generalized NLSE is characterized by a dynamical $O(2,1)$ symmetry in absence of the trap.

Consider the following Lagrangian in arbitrary $d+1$ dimensions,

$$
\mathcal{L}=i \psi^{*} \partial_{\tau} \psi-\frac{1}{2 m}|\boldsymbol{\nabla} \psi|^{2}-g V\left(\psi, \psi^{*}, \mathbf{r}\right) .
$$

The coupling constant $g$ has the inverse-mass dimension in the natural units with $c=\bar{h}=1$. The real potential $V$ does not depend on any dimensional coupling constant. This allows to have a scale-invariant theory. We demand the invariance of the action $\mathcal{A}=\int d \tau d^{d} \mathbf{r} \mathcal{L}$ under the following time-dependent transformations [四河],

$$
\begin{aligned}
& \mathbf{r} \rightarrow \mathbf{r}_{\mathbf{h}}=\dot{\tau}(t)^{-\frac{1}{2}} \mathbf{r}, \quad \tau \rightarrow t=t(\tau), \quad \dot{\tau}(t)=\frac{d \tau(t)}{d t}, \\
& \psi(\tau, \mathbf{r}) \rightarrow \psi_{h}\left(t, \mathbf{r}_{h}\right)=\dot{\tau}^{\frac{d}{4}} \exp \left(-i m \frac{\ddot{\tau}}{4 \dot{\tau}} r_{h}^{2}\right) \psi(\tau, \mathbf{r}),
\end{aligned}
$$

with the scale-factor $\tau$ given by,

$$
\tau(t)=\frac{\alpha t+\beta}{\gamma t+\delta}, \quad \alpha \delta-\beta \gamma=1 .
$$

Particular choices of $\tau(t)=t+\beta, \alpha^{2} t$, and $\frac{t}{1+\gamma t}$, correspond to time translation, dilation and special conformal transformation. The generators of these transformations ( as given below ) close under an $O(2,1)$ algebra. Although $V$ is restricted to have specific forms due to the requirement of the symmetry, one can still make infinitely many choices of it. It might be noted here, apart from its dependence on the condensate $\psi$, the potential can also be explicitly dependent on the space-coordinates. We keep $V$ arbitrary, but, consistent with the $O(2,1)$ symmetry in (11), unless mentioned otherwise.

Let us now introduce two moments $I_{1}$ and $I_{2}$ in terms of the density $\rho$ and the current $\mathbf{j}$ as,

$$
\begin{aligned}
& \rho(\tau, \mathbf{r})=\psi^{*} \psi, \quad \mathbf{j}(\tau, \mathbf{r})=-\frac{i}{2 m}\left(\psi^{*} \boldsymbol{\nabla} \psi-\psi \boldsymbol{\nabla} \psi^{*}\right), \\
& I_{1}(\tau)=\frac{m}{2} \int d^{d} \mathbf{r} r^{2} \rho, \quad I_{2}(\tau)=\frac{m}{2} \int d^{d} \mathbf{r} \mathbf{r} \cdot \mathbf{j} .
\end{aligned}
$$

We are dealing with a conservative system and the total number of particles $N(\tau)=\int d^{d} \mathbf{r} \rho$ is a constant of motion. The moment $I_{2}$ is related to the speed of the growth of the condensate. The moment $I_{1}$ describes the square of the width of the wave-packet [6]. This quantity plays the central role in the analysis of the collapse of the condensates of the NLSE with or without timeindependent harmonic trap [7 10]. It is also used in the context of the Bose-Einstein condensation(BEC) [11] to study the low energy excitations and in optics [12] to determine the beam parameter evolution. The dynamics of $I_{1}$, when the system (11) is immersed in an external timedependent harmonic trap, is the central subject of the investigation of this letter. We show that the dynamics of $I_{1}$ is universally determined by the same solvable Hill's equation, independent of the space-dimensionality, integrability and nature ( short-range, long-range, local, nonlocal, linear, non-linear ) of the interaction. The universality in the description of the dynamics of the width for this class of theory has been observed partially through a time-dependent variational analysis in [13]. We present here exact, analytical and complete treatment.

The system (1) has a dynamical $O(2,1)$ symmetry. The generators, the Hamiltonian $H$, the dilatation generator $D$ and the conformal generator $K$ are,

$$
\begin{aligned}
& H=\int d^{d} \mathbf{r}\left[\frac{1}{2 m}|\boldsymbol{\nabla} \psi|^{2}+g V\left(\psi, \psi^{*}, \mathbf{r}\right)\right], \\
& D=\tau H-I_{2} \\
& K=-\tau^{2} H+2 \tau D+I_{1} .
\end{aligned}
$$


These generators are constant in time and lead to the following equations,

$$
\frac{d H}{d \tau}=0, \quad \frac{d I_{1}}{d \tau}=2 I_{2}, \quad \frac{d I_{2}}{d \tau}=H .
$$

For time independent solutions, both $I_{1}$ and $I_{2}$ do not depend on $\tau$. As a consequence, the static solutions of a system with $O(2,1)$ symmetry carry zero energy [14]. We also note that $I_{2}=D=0$ and $K=I_{1}$ for static solutions of (11). Defining the width of the wave-packet, $X=\sqrt{I_{1}}$, it is easy to find a decoupled equation for $X$ from (6),

$$
\frac{d^{2} X}{d \tau^{2}}=\frac{Q}{X^{3}}, \quad Q=I_{1} H-I_{2}^{2}>0, \quad \frac{d Q}{d \tau}=0 .
$$

The constant of motion $Q$ is the Casimir operator of the $O(2,1)$ symmetry. Eq. (7) can be interpreted as the equation of motion of a particle moving in an inversesquare potential. Interestingly enough, this system also has a dynamical $O(2,1)$ symmetry. This reduced system of a particle in an inverse-square potential is a wellstudied problem and the solution is given by [4],

$$
X^{2}=(a+b \tau)^{2}+\frac{Q}{a^{2}} \tau^{2}
$$

where $a$ and $b$ are the integration constants.

Consider the time-dependent transformations in (2) with arbitrary scale-factor $\tau(t)$. This is no more symmetry transformations of (11) for general $\tau$. The action $\mathcal{A}$ is transformed into a new one, $\mathcal{A}_{h}=\int d t d^{d} \mathbf{r}_{h} \mathcal{L}_{h}$, containing a time-dependent harmonic trap. The new Lagrangian $\mathcal{L}_{h}$ now reads,

$$
\begin{aligned}
\mathcal{L}_{h} & =i \psi_{h}^{*} \partial_{t} \psi_{h}-\frac{1}{2 m}\left|\nabla_{h} \psi_{h}\right|^{2} \\
& -g V\left(\psi_{h}, \psi_{h}^{*}, \mathbf{r}_{h}\right)-\frac{1}{2} m \omega(t) r_{h}^{2}\left|\psi_{h}\right|^{2} .
\end{aligned}
$$

The time-dependent frequency $\omega(t)$ of the harmonic trap is determined by,

$$
\ddot{b}+\omega(t) b=0, \quad b(t)=\dot{\tau}^{-\frac{1}{2}} .
$$

Once the solution of the equation of motion of (11) is known, the same can be obtained for (9) by using the transformation (2) and the equation (10) or the vice versa. Equation (10) describes the motion of a particle in a time-dependent harmonic trap. For $\tau(t)=\frac{1}{\tan }\left(\omega_{0} t\right)$, it gives $\omega=\omega_{0}$. For the special choice of (3), the frequency $\omega$ obviously vanishes. The general solution of (10) for the physically relevant periodic $\omega(t)$ is well-known and will be discussed below.

The dynamical $O(2,1)$ symmetry of $\mathcal{L}$ is not present for $\mathcal{L}_{h}$. We replace $(\mathbf{r}, \psi, \tau)$ by $\left(\mathbf{r}_{h}, \psi_{h}, t\right)$ in the definition of $I_{1}, I_{2}$ and $H$ and denote the resulting expressions in terms of the 'curly form' of the associated variables.
Under the transformation (2), the equations (6) have the following form,

$$
\begin{aligned}
& \dot{\mathcal{I}}_{1}(t)=2 \mathcal{I}_{2}(t), \\
& \dot{\mathcal{I}}_{2}(t)=\mathcal{H}(t)-\omega(t) \mathcal{I}_{1}(t), \\
& \dot{\mathcal{H}}(t)=-2 \omega(t) \mathcal{I}_{2}(t) .
\end{aligned}
$$

Defining a new variable $\mathcal{X}(t)=\sqrt{\mathcal{I}_{1}(t)}$, it is easy to find a decoupled equation for $\mathcal{X}$,

$$
\begin{aligned}
& \ddot{\mathcal{X}}+\omega(t) \mathcal{X}=\frac{\mathcal{Q}}{\mathcal{X}^{3}}, \\
& \mathcal{Q}=\mathcal{I}_{1} \mathcal{H}-\mathcal{I}_{2}^{2}>0, \quad \dot{\mathcal{Q}}=0 .
\end{aligned}
$$

We have the surprising result that the dynamics of the width of the wave-packet of the system (9) is universally determined by the equation (12). This result is independent of the integrability of the model. We also have the freedom of choosing a large class of $V$ as long as the dynamical $O(2,1)$ symmetry in absence of the harmonic trap is maintained. The knowledge of the time-evolution of $\mathcal{X}$ allows us to determine the time-evolution of $\mathcal{H}$,

$$
\mathcal{H}=\dot{\mathcal{X}}^{2}+\frac{\mathcal{Q}}{\mathcal{X}^{2}}
$$

For the time-independent trap, $\omega(t)=\omega_{0}$, the Hamiltonian $H_{h}=\mathcal{H}+\omega_{0} \mathcal{I}_{1}$ corresponding to the Lagrangian $\mathcal{L}_{h}$ is a constant of motion. The Hamiltonian $H_{h}$ is related to the generator of the compact $S O(2)$ rotation of $S O(2,1)$.

Eq. (12) can be interpreted as that of a particle moving in a time-dependent harmonic trap and a inverse-square potential. Due to the underlying $O(2,1)$ symmetry in absence of the trap, this equation can be obtained directly from (7) through the use of the transformations (2). The dynamics of the width $\mathcal{X}$ can thus be constructed exactly from (8),

$$
\mathcal{X}(t)=b(t) X(\tau(t))
$$

with the knowledge of the scale-factors $\tau(t)$ and $b(t)$ from (10) for a particular choice of $\omega(t)$. We provide below a familiar form of solution of Eq. (12), since it appears in many branches of physics including the cylindrically symmetric two-dimensional NLSE [10]. The general solution of (12) is given by,

$$
\mathcal{X}^{2}(t)=u^{2}(t)+\frac{Q}{W^{2}} v^{2}(t), \quad W(t)=u \dot{v}-v \dot{u}
$$

where $u(t)$ and $v(t)$ are two independent solutions of the equation,

$$
\ddot{x}+\omega(t) x=0,
$$

satisfying $u\left(t_{0}\right)=\mathcal{X}\left(t_{0}\right), \dot{u}\left(t_{0}\right)=\dot{\mathcal{X}}\left(t_{0}\right), \dot{v}\left(t_{0}\right)=0$ and $v\left(t_{0}\right) \neq 0$. For periodic $\omega(t)$ with the period $T$, the above 
equation is known as the Hill's equation and is a textbook material 15. We just mention here the general stability criteria in terms of the quantity $\delta=|u(T)+\dot{v}(T)|$ with the normalization $\mathcal{X}(0)=0, \dot{\mathcal{X}}(0)=1$ and $v(0)=1$. The solution is stable for $\delta<2$, while it is unstable for $\delta>2$. We remark that the same stability criteria is valid for Eq. (10).

The central result of this letter is contained in (12). Although our main concern in this letter is on NLSE, we remark that the same result is true for a class of linear Schrödinger equations with Calogero-type inverse-square interaction in arbitrary dimension 16. An example of arbitrary $d$ dimensional non-linear potential consistent with $O(2,1)$ symmetry is given by,

$$
V\left(\psi, \psi^{*}, \mathbf{r}\right)=\int d^{d} \mathbf{r}^{\prime} \psi^{*}\left(\mathbf{r}^{\prime}\right) U\left(\mathbf{r}-\mathbf{r}^{\prime}\right) \psi\left(\mathbf{r}^{\prime}\right)|\psi(\mathbf{r})|^{2},
$$

with $U(r)$ having the following scaling property. For $\mathbf{r} \rightarrow$ $\epsilon \mathbf{r}, U(\mathbf{r}) \rightarrow U(\epsilon \mathbf{r})=\epsilon^{-2} U(\mathbf{r})$. We now discuss a few specific examples of NLSE with different choices of $V$ which are relevant in the contemporary literature.

$B E C$ in $d=1, g>0, V=|\psi|^{6}$ : The Gross-Pitaevskii equation (GPE) describing the repulsive Bose-Einstein condensates trapped in a time-dependent harmonic trap in one dimension can be obtained from the Lagrangian (9) 17. Exact soliton solutions of the GPE equation have been obtained in absence of the trap [17,18]. Only approximate or numerical results are known, when the time-independent harmonic trap is included 17 .

Following our analysis, the exact solutions of (9) can be obtained from those of (1) by simply using the transformation (2) and the equation (10) determining the timedependent scale-factor $\tau$ for a particular choice of the $\omega(t)$. We consider the case of time-independent trap with $\omega(t)=\omega_{0}$ and choose $g=\frac{\pi^{2}}{6 m}$. Define the following dimensionless variables,

$$
\bar{x}_{h}=\pi \psi_{0}^{2} x_{h}, \quad \bar{t}=\frac{\pi^{2} \psi_{0}^{4}}{m} t, \quad \bar{\psi}_{h}=\frac{\psi_{h}}{\psi_{0}}, \quad \bar{\omega}_{0}=\frac{m \omega_{0}}{\pi^{4} \phi_{0}^{8}},
$$

where $\psi_{0}^{2}$, the asymptotic value of the density, is related to the chemical potential $\mu$ by, $\psi_{0}^{2}=\frac{\sqrt{2 m \mu}}{\pi}$. The exact solution for $\bar{\psi}_{h}$ is,

$$
\begin{aligned}
\bar{\psi}_{h}= & \frac{1}{\cos \left(\bar{\omega}_{0} \bar{t}\right)} \exp \left(-\frac{i \bar{\omega}_{0}}{2} \tan \left(\bar{\omega}_{0} \bar{t}\right) \bar{x}_{h}^{2}-\frac{i}{\bar{\omega}_{0}} \tan \left(\bar{\omega}_{0} \bar{t}\right)\right) \\
& \times\left[\frac{\cosh \left[\frac{2 y}{\cos \left(\bar{\omega}_{0} t\right)}\right]-1}{\cosh \left[\frac{2 y}{\cos \left(\bar{\omega}_{0} t\right)}\right]+2}\right]^{\frac{1}{2}}
\end{aligned}
$$

In the limit $\bar{\omega}_{0} \rightarrow 0$, the solution for the system without the trap is recovered [17]. Without loss of any generality, we are choosing $\beta=0$ in Eq. (12) of 17.

BEC in $d=2, V=|\psi|^{4}$ : We get the GPE describing two dimensional BEC in a time-dependent trap from the Lagrangian (9). No exact solution of this GPE with or without the trap is known. However, this is a well-studied system and many of the dynamical properties are already known 19,20,10]. We concentrate here on the rotating BEC and present some new interesting results. Consider a further time-dependent rotation in (9) [5],

$$
t \rightarrow \tilde{t}=t, \quad \mathbf{r}_{h} \rightarrow \tilde{\mathbf{r}}_{h}=\left(\begin{array}{cc}
\cos f(t) & \operatorname{sinf}(t) \\
-\operatorname{sinf}(t) & \cos f(t)
\end{array}\right) \mathbf{r}_{h} .
$$

This transforms $\mathcal{A}_{h}$ to $\tilde{\mathcal{A}}_{h}=\int d \tilde{t} d^{d} \tilde{\mathbf{r}}_{h} \tilde{\mathcal{L}}_{h}$ containing an additional term proportional to the $z$ component of the angular momentum with the coefficient given by a timedependent frequency. In particular, the new $\tilde{\mathcal{L}}_{h}$ is given by,

$$
\begin{aligned}
\tilde{\mathcal{L}}_{h} & =i \psi_{h}^{*} \partial_{\tilde{t}} \psi_{h}-\frac{1}{2 m}\left|\tilde{\nabla}_{h} \psi_{h}\right|^{2}-g V\left(\psi_{h}, \psi_{h}^{*}, \tilde{\mathbf{r}}_{h}\right) \\
& -\frac{1}{2} m \omega(t) \tilde{r}_{h}^{2}\left|\psi_{h}\right|^{2}-\dot{f} \psi_{h}^{*} L_{z} \psi_{h}, \\
L_{z} & =-i\left(\tilde{x}_{h} \frac{\partial}{\partial \tilde{y}_{h}}-\tilde{y}_{h} \frac{\partial}{\partial \tilde{x}_{h}}\right),
\end{aligned}
$$

where $\tilde{x}_{h}$ and $\tilde{y}_{h}$ are the components of the two dimensional vector $\tilde{\mathbf{r}}_{h}$. This is the Lagrangian for rotating BEC in an external time-dependent isotropic trap in two dimensions 21]. Interestingly, once the solution of the equation of motion of (11) is known, the same can be obtained for (21), using the equations (22, (10) and (20), or vice versa. Moreover, under the transformation (20), the set of equations in (11) remains the same in terms of the new variables $\tilde{t}$ and $\tilde{\mathbf{r}}_{h}$. Thus, the dynamics of the width $\mathcal{X}(\tilde{t})$ of (21) is again universally determined by the equation (12). The introduction of the last term in (21) does not change the dynamical properties of the width. There may be dynamic instabilities solely due to the rapid fluctuations in the phase of the condensate during the evolution in time. However, it is obvious from the definition of $I_{1}$ that such instabilities do not show up in the evolution of the width.

Gauged NLSE: We now show that the dynamics of the width remains unchanged even if the non-trivial gaugefields are introduced in (11) maintaining the $O(2,1)$ symmetry. Consider a Lagrangian in $2+1$ dimensions with the gauge-fields $\left(A_{0}, \mathbf{A}\right)$ and the matter-field $\psi$,

$$
\begin{aligned}
\mathcal{L}_{g} & =i \psi^{*}\left(\partial_{\tau}-i A_{0}\right) \psi-\frac{1}{2 m}|(\boldsymbol{\nabla}-i \mathbf{A}) \psi|^{2} \\
& -g V\left(\psi, \psi^{*}, \mathbf{r}\right)+\frac{\kappa}{4} \epsilon^{\mu \nu \lambda} F_{\mu \nu} A_{\lambda},
\end{aligned}
$$

where the last term is the Chern-Simons term. The moment $I_{1}$ for this non-relativistic Chern-Simons(CS) theory can be interpreted as the width of the soliton or alternatively as the quadrupole moment. For $V=\frac{1}{2}|\psi|^{4}$, this is the Jackiw-Pi model describing gauged NLSE [22]. This is relevant in theories with anyons and in the quantum Hall effect 222 24. The Jackiw-Pi model is exactly solvable at the self-dual point, $g=\frac{1}{m|\kappa|}$. Our result is 
valid for arbitrary $V$ maintaining $O(2,1)$ symmetry. For the particular case of Jackiw-Pi model, the importance of our result lies at all non-self-dual points, where the model is not integrable. The Hamiltonian is given by,

$$
H=\int d^{2} \mathbf{r}\left[\frac{1}{2 m}|(\boldsymbol{\nabla}-i \mathbf{A}) \psi|^{2}+g V\left(\psi, \psi^{*}, \mathbf{r}\right)\right] \text {. }
$$

The CS term being a topological term do not contribute to the Hamiltonian. The generators $D$ and $K$ have the same expressions as in (5), with the partial derivative in the expression of the current $\mathbf{j}$ in the definition of the moment $I_{2}$ replaced by the respective covariant derivative. The treatment is now identical to the case without the gauge-fields. The same transformations (2) with $d=2$ and the gauge-fields transforming accordingly,

$$
\begin{aligned}
& A_{\mu}^{h}\left(t, \mathbf{r}_{h}\right)=\frac{\partial x^{\nu}}{\partial x_{h}^{\mu}} A_{\nu}(\tau, \mathbf{r}), \\
& x^{\mu}=(\tau, \mathbf{r}), \quad A^{\mu}=\left(A_{0}, \mathbf{A}\right), \quad \mu=0,1,2,
\end{aligned}
$$

introduce a time-dependent harmonic trap [22 24]. The width of the soliton of this new Lagrangian is again universally determined by (12). Interestingly, the introduction of the gauge-fields and the nontrivial CS term to the usual two dimensional NLSE does not change the dynamics of $\mathcal{X}$. A comment is in order at this point. It is known that Eq.(12) admits parametric resonances. Thus, the solitons of the non-relativistic CS theory should exhibit the same phenomenon. This provides an example of exact, extended parametric resonance in a gauge theory with the nontrivial CS term.

In conclusion, we have shown that the width of the wave-packet of a class of generalized NLSE is universally determined by the same Hill's equation. This class of NLSE is characterized by a dynamical $O(2,1)$ symmetry in absence of the trap. The result is so robust that it is independent of, (i) the space dimensionality, (ii) the integrability of the model, and (iii) short-range, long-range, local, non-local, linear or non-linear nature of the manybody interaction. This result persists with its full generality even when the gauge-fields are introduced maintaining the dynamical $O(2,1)$ symmetry. The later example allows us to study an exact parametric resonance in a theory with the nontrivial gauge fields. Special cases of this class of generalized NLSE are relevant in BEC and in non-relativistic Chern-Simons theory. It would be nice to see the importance of this class of NLSE in many more physical systems.

\section{ACKNOWLEDGMENTS}

This work is supported by a fellowship (P99231) of the JSPS. I would like to thank T. Deguchi and T. K. Ghosh for their continuous interest in this work and valuable comments on the manuscript.
[1] V. E. Zakharov and A. B. Shabat, Sov. Phys. JETP 34, 62 (1972); R. Camassa, J. M. Hyman and B. P. Luce, Physica 123D, 1 (1998).

[2] A. Hasegawa and F. Tappert, Appl. Phys. Lett. 23, 142 (1973); 23, 171 (1973).

[3] L. F. Mollenauer, R. H. Stolen and J. P. Gordan, Phys. Rev. Lett. 45, 1095 (1980).

[4] V. de Alfaro, S. Fubini and G. Furlan, Nuvo Cimento A34, 569 (1976); R. Jackiw, Ann. Phys. (N.Y.) 129, 183(1980); 201, 83 (1990).

[5] S. Takagi, Prog. Theor. Phys. 84, 1019 (1990); 85, 463 (1991); 85, 723 (1991).

[6] We are using the phrase 'width of the wave-packet' following Ref. 10]. It may be more appropriate for some specific cases to identify $I_{1}$ with a different physical quantity instead of the width. The central result, the exact and the universal description of the dynamics of the moment $I_{1}$, is independent of any such change in the nomenclature.

[7] V. E. Zakharov, Sov. Phys. JETP 35, 908 (1972); V. E. Zakharov and V. S. Synakh, Sov. Phys. JETP 41, 465 (1975); M. I. Weinstein, Commun. Math. Phys. 87, 567 (1983).

[8] T. Tsurumi and M. Wadati, J. Phys. Soc. Japan 66, 3031 (1997); ibid 66, 3035 (1997); M. Wadati and T. Tsurumi, Phys. Lett. A 247, 287 (1998); T. Tsurumi, H. Morise and M. Wadati, Int. Jour. Mod. Phys. B 14, 655 (2000), cond-mat/9912470.

[9] A. V. Rybin, G. G. Varzugin, M. Lindberg, J. Timonen and R. K. Bullough, Phys. Rev. E 62, 6224 (2000), condmat/0001059.

[10] J. J. Garcia-Ripoll and V. M. Perez-Garcia, Phys. Rev. Lett. 83, 1715 (1999); patt-sol/9904006.

[11] S. Stringari, Phys. Rev. Lett. 77, 2360 (1996); V. M. Perez-Garcia et. al., Phys. Rev. Lett. 77, 5320 (1996); F. Dalfovo, S. Giorgini, L. P. Pitaevskii and S. Stringari, Rev. Mod. Phys. 71463 (1999), cond-mat/9806038.

[12] S. N. Vlasov, V. A. Petrischev and V. I. Talanov, Radiophys. Quantum Electron. 14, 1062 (1971); V. M. PerezGarcia et. al., J. Opt. B : Quantum Semiclass. Opt. 2, 353358 (2000).

[13] T. K. Ghosh, Phys. Lett. A 285, 222 (2001), condmat/0012188.

[14] D. Freedman and A. Newell (unpublished).

[15] V. I. Arnold, Mathematical Methods of Classical Mechanics, Second Edition ( Springer-Verlag, New York, 1989 ).

[16] Pijush K. Ghosh ( unpublished ).

[17] E. B. Kolomeisky, T. J. Newman, J. P. Straley and X. Qi, Phys. Rev. Lett. 85, 1146 (2000).

[18] R. K. Bhaduri, S. Ghosh, M. V. N. Murthy and D. Sen, J. Phys. A: Math. Gen. 34, 6553 (2001), condmat/0010075.

[19] Yu. Kagan, E. L. Surkov and G. V. Shlyapnikov, Phys. Rev. A 54, R1753 (1996).

[20] L. P. Pitaevskii and A. Rosch, Phys. Rev. A 55, R835 (1997), cond-mat/9608135. 
[21] A. L. Fetter and A. A. Svidzinsky, cond-mat/0102003.

[22] R. Jackiw and S.-Y. Pi, Phys. Rev. Lett. 64, 2969 (1990); 66, 2682 (1991); Phys. Rev. D 42, 3500 (1990); D 44, 2524 (1991).

[23] Z. F. Ezawa, M. Hota and A. Iwazaki, Phys. Rev. Lett. 67, 411 (1991).

[24] Pijush K. Ghosh, Phys. Rev. D 53, 2248 (1996), hepth/9503199. 